\title{
A convergent highly stereoselective preparation of the C12-C24 fragment of macrolactin A
}

Carlo Bonini, ${ }^{* \dagger}$ Lucia Chiummiento, ${ }^{\dagger}$ Maddalena Pullez, ${ }^{\dagger}$ Guy Solladiè, *** Françoise Colobert ***

Dipartimento di Chimica, Università degli Studi della Basilicata, via N. Sauro 85, 85100 Potenza, Italy and Laboratoire de Stéréochimie associé au CNRS, Université Louis Pasteur, ECPM, 25 rue

Becquerel, 67087 Strasbourg Cedex 2, France

bonini@unibas.it

Table of contents:

Experimental section

S2-S3

${ }^{1} \mathrm{H}$ NMR Spectrum of $\mathbf{2}$

${ }^{13} \mathrm{C}$ NMR spectrum of $\mathbf{2}$

S5

COSY NMR spectrum of 2

S6 


\section{Experimental section}

\section{General methods}

Unless otherwise specified, materials were purchased from commercial suppliers and used without further purification. THF, toluene, diethyl ether were distilled from sodium/benzophenone ketyl immediately before use. Dichloromethane was distilled from $\mathrm{P}_{2} \mathrm{O}_{5}$. Acetonitrile was distilled from calcium hydride. Methanol was distilled from magnesium and iodine. Triethylamine and pyridine were distilled from calcium hydride and stored over potassium hydroxide. DMF was freshly distilled and stored over $4 \AA$ sieves. Moisture-sensitive reactions were conducted in oven- or flamedried glassware under an argon atmosphere. All reactions were magnetically stirred and monitored by thin-layer chromatography using precoated silica gel $\left(60 \mathrm{~F}_{254}\right)$ plates. Column chromatography was performed with the indicated solvents using silica gel-60 •. Mass spectra were obtained by GC/MS with electron impact ionization. NMR spectra were recorded in $\mathrm{CDCl}_{3}$ solution at room temperature $\left({ }^{1} \mathrm{H}\right.$ at 200, 300 and $400 \mathrm{MHz}$ and ${ }^{13} \mathrm{C}$ at 50, 75 and $100 \mathrm{MHz}$, respectively). Chemical shifts $(\delta)$ were expressed in ppm and coupling constant $(J)$ in hertz. Optical rotations were determined operating at the sodium D line at $25{ }^{\circ} \mathrm{C}$. HPLC analyses were conducted using Chiral OJ column with UV detection at $245 \mathrm{~nm}$.

(4R)-1-(2,2-Dimethyl-[1,3]dioxolan-4-yl)-prop-2-yne-1-ol (24). Compound 24 was obtained as a mixture 54:76 of diastereomers from $\mathbf{2 3}$ following the same protocol as for compound 29. Purification on silica gel $\left(\mathrm{Et}_{2} \mathrm{O} /\right.$ hexane 1:1) provided compound $\mathbf{2 4}$ as a colorless oil (83\% yield). $R_{f}$ $=0.76\left(\right.$ EtOAc/hexane 7:3); ${ }^{1} \mathrm{H}$ NMR $\left(400 \mathrm{MHz}, \mathrm{CDCl}_{3}\right) \delta 1.35(\mathrm{~s}, 3 \mathrm{H}), 1.44(\mathrm{~s}, 3 \mathrm{H}), 2.48(\mathrm{~d}, J=2$ $\mathrm{Hz}, 1 \mathrm{H}), 2.70(\mathrm{~d}, J=5.2 \mathrm{~Hz}, 1 \mathrm{H}), 3.02(\mathrm{~d}, J=5 \mathrm{~Hz}, 1 \mathrm{H}), 3.89-4.41(\mathrm{~m}, 4 \mathrm{H})$.

(4R)-1-(2,2-Dimethyl-[1,3]dioxolan-4-yl)-5-methyl-hex-2-yne-1,4-diol (25). Compound 25 was obtained as a mixture of diastereomers from $\mathbf{2 4}$ following the same protocol as for compound $\mathbf{3 0 .}$ Purification on silica gel (EtOAc/hexane 1:1) provided compounds $\mathbf{2 4}$ as a yellow oil (80\% yield). $R_{f}=0.49\left(\right.$ EtOAc/hexane 7:3); ${ }^{1} \mathrm{H}$ NMR $\left(200 \mathrm{MHz}, \mathrm{CDCl}_{3}\right) \delta 0.96(\mathrm{~d}, J=4 \mathrm{~Hz}, 3 \mathrm{H}), 1.00(\mathrm{~d}, J=3.6$ Hz, 3H), 1.87 (sept., 1H), 2.52 (bs, 1H), 2.77 and 2.92 (bs, 1H), 3.94-4.51 (m, 5H). EI-MS m/z: $\mathrm{M}^{+}-$ 15, (100), 101. 
(4R)-1-(2,2-Dimethyl-[1,3] dioxolan-4-yl)-5-methyl-hex-2-ene-1,4-diol (26). Compound 26 was obtained, as a mixture of diastereomers, from $\mathbf{2 5}$ following the same protocol as for compound $\mathbf{3 1}$. Purification on silica gel ( $\mathrm{Et}_{2} \mathrm{O} /$ hexane 1:1) provided the compound $\mathbf{2 6}$ as a yellow oil in $70 \%$ yield. $R_{f}=0.46$ and $0.39\left(\mathrm{Et}_{2} \mathrm{O} /\right.$ hexane 8:2); ${ }^{1} \mathrm{H} \mathrm{NMR}\left(200 \mathrm{MHz}, \mathrm{CDCl}_{3}\right) \delta 0.87(\mathrm{~d}, J=6.9 \mathrm{~Hz}, 3 \mathrm{H}), 0.97$ and $0.93(\mathrm{~d}, J=6.6 \mathrm{~Hz}, 3 \mathrm{H}), 1.32$ and $1.35(\mathrm{~s}, 3 \mathrm{H}), 1.41$ and $1.45(\mathrm{~s}, 3 \mathrm{H}), 1.69$ and 1.72 (sept., $1 \mathrm{H})$, 2.28 (bs, 1H), 2.57 (bs, 2H), 2.65 (bs, 1H), 3.76-4.14 (m, 4H), $4.41(\mathrm{~m}, 1 \mathrm{H}), 4.50(\mathrm{~m}, 1 \mathrm{H}), 5.42-$ $5.56(\mathrm{~m}, 2 \mathrm{H})$.

(4R)-benzoic acid 4-benzoyloxy-1-(2,2-dimethyl-[1,3]dioxolan-4-yl)-5-methyl-hex-2-enyl ester (27). Compound 27 was obtained as a mixture of diastereomers from $\mathbf{2 6}$ following the same protocol as for compound 32. Purification on silica gel (hexane/Et ${ }_{2} \mathrm{O} 9: 1$ ) provided the compound 27 as a colorless oil (94\% yield). $R_{f}=0.76$ (hexane/Et $\left.\mathrm{O}_{2} 8: 2\right) ;{ }^{1} \mathrm{H} \mathrm{NMR}\left(200 \mathrm{MHz}, \mathrm{CDCl}_{3}\right) \delta 1.01$ $(\mathrm{m}, 6 \mathrm{H}), 1.37(\mathrm{~s}, 3 \mathrm{H}), 1.51(\mathrm{~s}, 3 \mathrm{H}), 2.07(\mathrm{~m}, 1 \mathrm{H}), 3.92-4.17(\mathrm{~m}, 2 \mathrm{H}), 4.40-4.59(\mathrm{~m}, 1 \mathrm{H}), 5.79(\mathrm{~m}$, $3 \mathrm{H}), 6.21(\mathrm{~m}, 1 \mathrm{H}), 7.40-7.60(\mathrm{~m}, 6 \mathrm{H}), 8.02-8.12(\mathrm{~m}, 4 \mathrm{H})$.

(4R,1E,3E)-2,2-Dimethyl-4-(5-methyl-hexa-1,3-dienyl)-[1,3]dioxolane (28). Compound 28 was obtained, as a single stereoisomer, from 27 following the same protocol as for compound 2. Purification on silica gel (hexane/Et $\mathrm{O}_{2}$ 9:1) provided the compound 27 as a colorless oil (90\% yield). $R_{f}=0.47$ (hexane/Et $\left.2 \mathrm{O} 9: 1\right) ;{ }^{1} \mathrm{H}$ NMR $\left(200 \mathrm{MHz}, \mathrm{CDCl}_{3}\right) \delta 0.98(\mathrm{~s}, 3 \mathrm{H}), 1.01(\mathrm{~s}, 3 \mathrm{H}), 1.39$ (s, $3 \mathrm{H}), 1.43$ (s, 3H), 2.34 (sept., $1 \mathrm{H}$ ), 3.56 and 4.08 (AB part of an ABX system, $J_{\mathrm{AB}}=8.3 \mathrm{~Hz}, J_{\mathrm{AX}}=7.9$ $\left.\mathrm{Hz}, J_{\mathrm{BX}}=6.3 \mathrm{~Hz}, 2 \mathrm{H}\right), 4.52(\mathrm{~m}, \mathrm{X}$ part of an $\mathrm{ABX}$ system, $1 \mathrm{H}), 5.53(\mathrm{dd}, J=15 \mathrm{~Hz}, J=8 \mathrm{~Hz}, 1 \mathrm{H})$, $5.70(\mathrm{dd}, J=15 \mathrm{~Hz}, J=7.6 \mathrm{~Hz}, 1 \mathrm{H}), 6.00(\mathrm{dd}, J=15 \mathrm{~Hz}, J=10 \mathrm{~Hz}, 1 \mathrm{H}), 6.27(\mathrm{dd}, J=15 \mathrm{~Hz}, J=$ $10 \mathrm{~Hz}, 1 \mathrm{H}) ;{ }^{13} \mathrm{C}$ NMR $\left(50 \mathrm{MHz}, \mathrm{CDCl}_{3}\right) \delta 22.17,25.93,26.71,29.67,31.06,69.49,73.00,102.30$, $126.14,127.53,134.30,143.47$. 


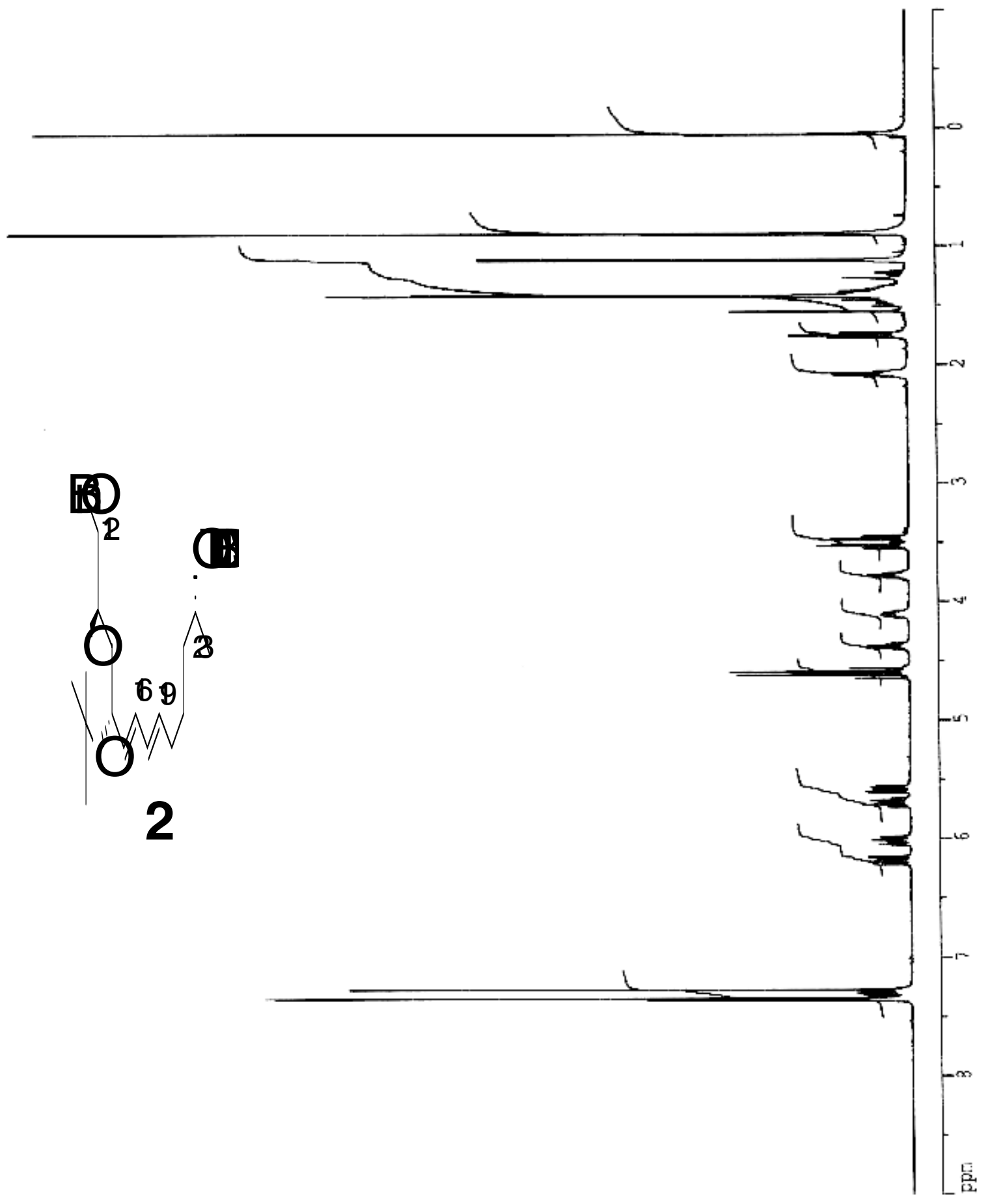




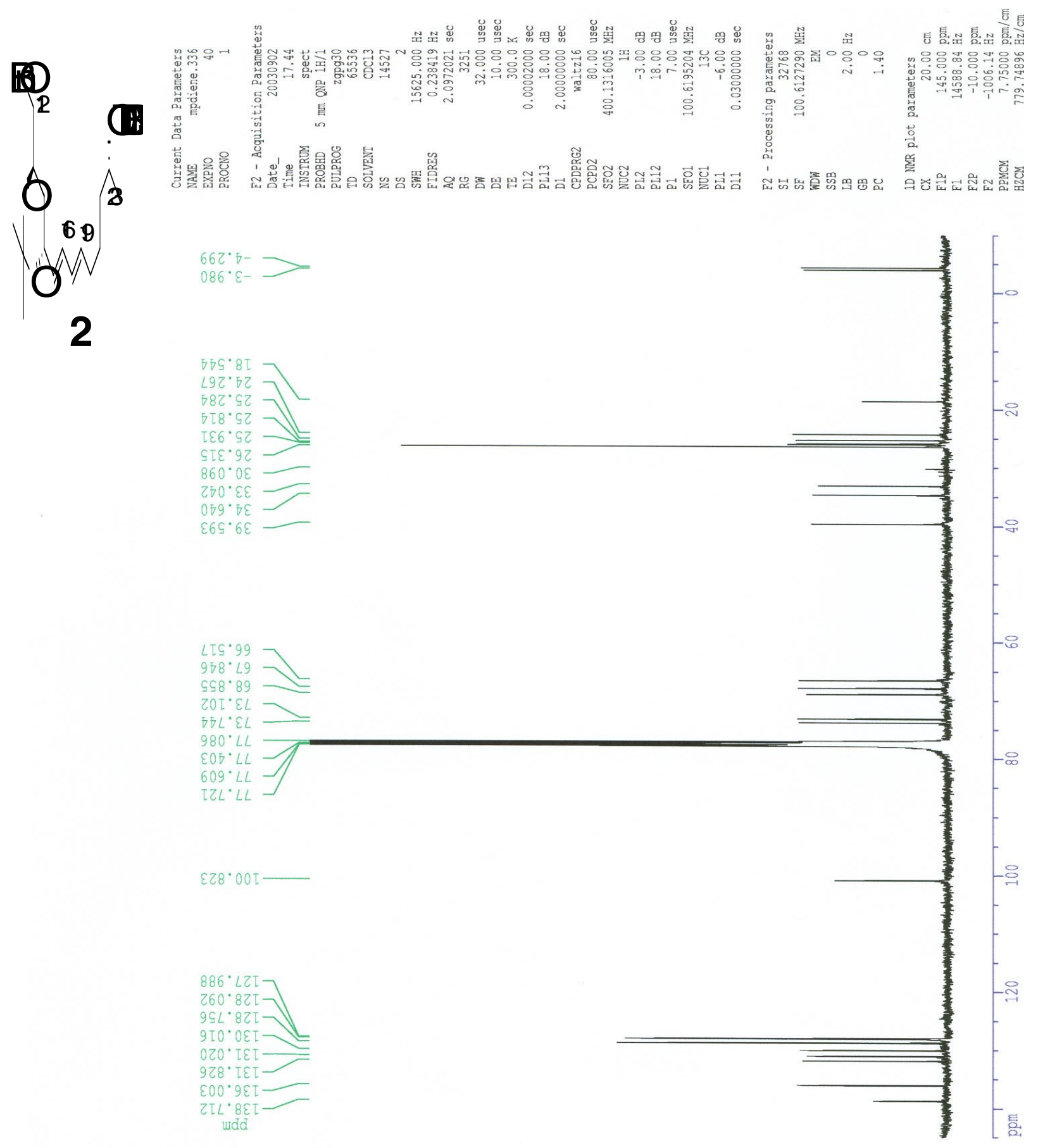




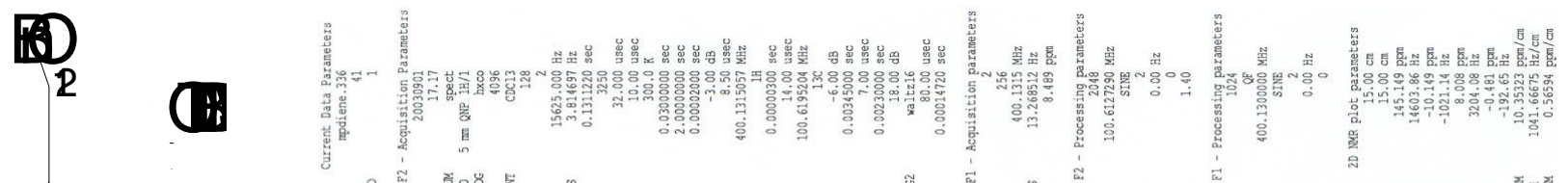

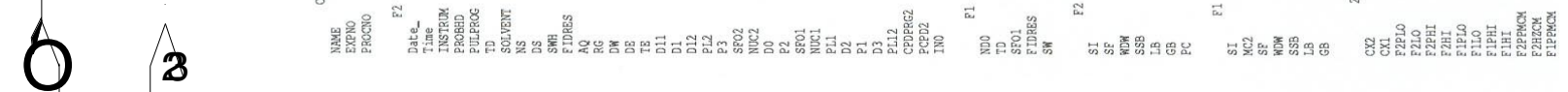
19

2
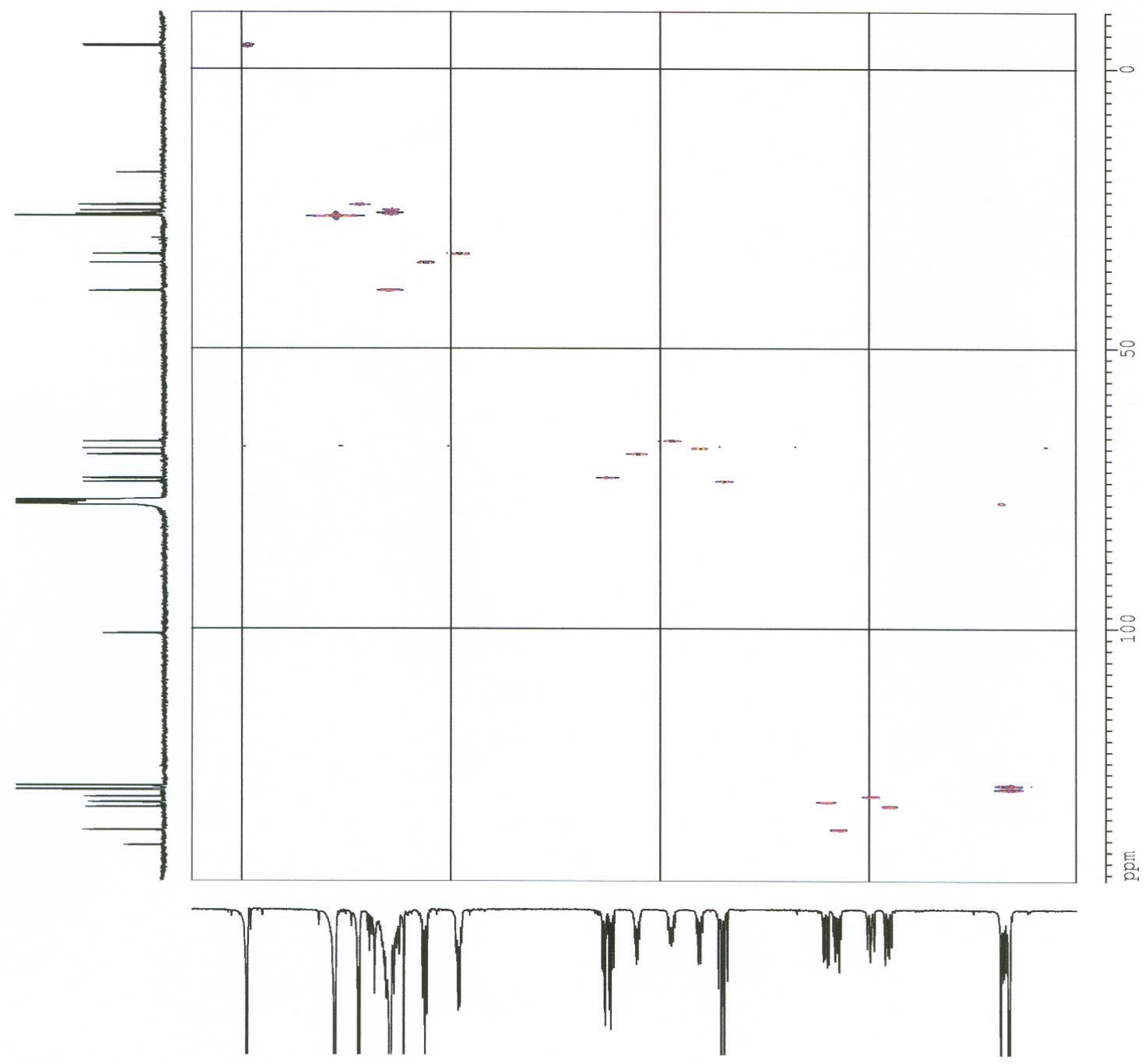